\title{
Sustainable effects of a low-threshold physical activity intervention on health-related quality of life in residential aged care
}

This article was published in the following Dove Press journal:

Clinical Interventions in Aging

3 November 2014

Number of times this article has been viewed

\section{Viktoria Quehenberger Martin Cichocki \\ Karl Krajic}

Health promoting Long term Care, Ludwig Boltzmann Institut Health Promotion Research, Vienna, Austria
Correspondence: Viktoria Quehenberger Ludwig Boltzmann Institut Health Promotion Research, Untere Donaustrasse 47, 3 floor, 1020 Vienna, Austria

Tel +43 | 2|2| 4934 |

Email viktoria.quehenberger@lbihpr.lbg. ac.at
Background: Mobility is a main issue for health-related quality of life in old age. There is evidence for effects of physical activity (PA) interventions on several dimensions of health for the aged and also, some specific evidence for vulnerable populations, like residents of residential aged care. Research on low-threshold PA interventions for users of residential aged care and documentation of their sustainability are scarce. "Low threshold" implies moderate demands on the qualification of trainers and low frequency of conduct, implying low demands on the health status and discipline of users. Yet the investigation of low-threshold interventions in residential aged care seems important as they might foster participation of users and implementation in everyday routines of provider organizations. An initial study (October 2011 to June 2012) had found intervention effects on health-related quality of life. The objective of this study was to examine sustainability of the effects of a low-threshold PA intervention on health-related quality of life in residential aged care.

Methods: Data collection took place in three residential aged care homes in Vienna, Austria. At 1-year follow-up (June 2013), participants from the intervention group were interviewed using a standardized questionnaire. Using general mixed linear models and Friedman tests followed by paired $t$ - and Wilcoxon signed-rank tests, we compared outcome measures at follow-up with measures obtained at baseline and at the end of the intervention.

Results: At the 1-year follow-up assessment, participants' (mean age 84.7 years; $89.7 \%$ female) subjective health status was still significantly increased, equaling a small sustainable intervention effect (Cohen's $d=0.38, P=0.02$ ). In comparison with baseline, a significant decline of reported pain/discomfort $(P=0.047)$ was found. Regarding the subdimensions of health-related quality of life, favorable trends could be observed.

Conclusion: The study indicates that effects of a low-threshold PA intervention on healthrelated quality of life in residential aged care can be sustainable. Addressing hindering factors like poor health status and implementing proactive support and individualization of the program to enable PA for residents might foster sustainability of effects.

Keywords: exercise group, long term care, effectiveness, follow-up, highly aged

\section{Introduction}

Physical activity (PA) and PA interventions in the aged have become important research topics over the last decades; yet a rather underresearched issue in this context is the sustainability of effects of these interventions. This holds true even more for users of residential aged care, who have rather high levels of morbidity and reduced functional health. Low-threshold PA interventions in residential aged care (ie, implying a combination of moderate demands on the qualification of trainers and low frequency of conduct) have hardly been investigated. Yet, research on these 
kinds of interventions seems important as they imply low demands on the health status and discipline of users and ask for rather little additional investment from provider organizations. The present study aimed to investigate the sustainability of effects of a low-threshold PA intervention in residential aged care.

\section{PA behavior and PA interventions}

A current review of international literature finds users of aged care to be a highly vulnerable and fragile group. Among this population, there is a high prevalence of chronic diseases and permanent physical and cognitive functional impairments. ${ }^{1}$ A study among US nursing homes found that prevalence of dementia among newly admitted residents was $48.2 \%{ }^{2}$ In addition, comparison of community-dwelling and institutionalized elderly without cognitive impairment showed that institutionalized elderly had significantly worse scores regarding functional ability, depression, satisfaction with life, health-related quality of life (as measured by the EQ-5D ${ }^{\mathrm{TM}}$ Index), and loneliness. ${ }^{3}$

An important determinant for health status and PA behavior of aged (60-79 years) and highly aged ( $80+$ years) persons is the aging process, which is associated with loss of functional and physiological health. Behavioral factors, like a sedentary lifestyle, are regarded as factors exacerbating various diseases. ${ }^{4}$ Yet a sedentary lifestyle is a widespread phenomenon among the aged and highly aged. ${ }^{5}$ Common strategies to counteract this trend and to enhance PA behavior are exercise programs. As for residents in long-term care, there is little research on participation in exercise programs. A Canadian study stated that $98 \%$ of nursing homes provided some kind of exercise and activity program; nevertheless, only $10 \%-15 \%$ of residents participated in these programs. ${ }^{6}$ A review indicates that barriers and facilitating factors for PA in the institutionalized aged and highly aged mainly relate to health; expectations of possible prevention of future health decline can be a supportive factor, while the level of perceived pain or changing health status can have a negative influence. Another frequently mentioned facilitating factor for PA is social support (by care staff, spouse, relatives, etc). ${ }^{7}$ A recent study on adherence rates of older women to strength training and aerobic exercise found worsening health status to be no significant predictor of poor adherence. Yet "changing health status" and "pain" was the most cited reason for missed sessions among nonadherents. ${ }^{8}$ It is likely that poor health status, as a barrier to PA participation, is more prominent in vulnerable groups, like residents of long-term care.
Positive effects of PA on physical, psychological, as well as functional dimensions of health have been demonstrated for the aged and even for institutionalized persons, including frail older persons.

Aged individuals with a high level of PA have a much lower risk of mortality, age-related morbidity, and loss of function. ${ }^{9}$ There are various positive effects of PA on physical health, including cardiovascular health. ${ }^{10} \mathrm{PA}$ is also known to have an effect in alleviating chronic pain. ${ }^{11}$ An increased level of PA is associated with higher levels of cognitive functioning and reduced cognitive decline. ${ }^{12}$

With respect to the psychological dimensions of the health of the aged, there is evidence that PA can cause significant improvements on overall well-being in the aged and on the quality of life of institutionalized older adults. ${ }^{13-15}$

Regarding the functional dimensions of health, in older adults, a sedentary lifestyle is associated with a decrease in physical functioning. ${ }^{9}$ As for institutionalized older persons and frail older persons, there is evidence for training effects on physical fitness, functional performance, and performance of activities of daily living. ${ }^{14,16}$

\section{Sustainability of effects of PA interventions}

There is evidence for various health improvements caused by PA interventions in the group of aged persons, yet the sustainability of these effects is rarely investigated. In general, one can distinguish two main goals of sustainability studies, either measuring the sustainability of effects of PA interventions on health status or investigating the behavioral change of participants as a relevant determinant of health status. This paper investigated the sustainability of effects on health status, due to the focus of an initial study (discussed below) on the health effects of PA interventions.

Review of the literature on the sustainability of health effects of PA interventions in the aged shows that interventions have been very heterogeneous. Studies vary in several dimensions:

- Population (community dwelling vs institutionalized, relatively healthy vs rather frail, prevalence of specific diseases, etc)

- Content of the program (aiming at strength, resistance, or balance, multicomponent, etc)

- Mode of delivery (activity advice, telephone follow-up, lifestyle counseling, exercise groups, etc)

- Frequency of units (from once a month up to approximately three or more times per week) 
- Duration of the program as well as time span between post- and follow-up assessments

- Outcome dimensions (objective vs subjective) and measurements (eg, EQ-5D vs Short Form $36^{\circledR}$ Health Survey; balance measured by tandem stance vs one-leg stance, etc).

The meta-analysis by Netz et al underlines the complex interaction of PA and psychological well-being in advanced age. The reviewed studies varied in duration (1-52 weeks of exercise), frequency ( $0.5-5$ sessions per week), and length (30-210 minutes per exercise session). Although they found strong evidence of the effectiveness of PA on psychological well-being, they also could show that results varied according to the outcome measure, duration, frequency, and length of sessions as well as their combination. ${ }^{13}$

Due to this heterogeneity and an unstandardized way in which these factors have been combined in available studies, it is difficult to draw general conclusions about the sustainability of effects. The following analysis discusses some examples, focusing on cases relevant for our specific intervention (ie, interventions involving exercise groups), and distinguishes between community-dwelling and institutionalized aged persons. Due to our interest in low-threshold interventions, we distinguish between low (= once per week), medium (= twice per week), and high exercise frequency (= three or more times per week).

\section{Community-dwelling aged}

With regard to high-frequency interventions in the community-dwelling aged, Pollock et al found effects were no longer clear 4 months after trial completion. ${ }^{17}$ In contrast, a multicomponent exercise program showed maintenance of significant improvements in balance and mobility at the 1-year follow-up visit, while improvements in strength vanished. $^{18}$

As for PA interventions with moderate frequency, Zech et al found an improvement in functional performance, but it was no longer evident at 24 and 36 weeks of follow-up. ${ }^{19}$ Another study compared exercise programs (eg, strength and balance, fitness, and multifaceted activity) and found improved mobility was sustainable for the fitness group at the 1-year mark but not at the 2-year follow-up visit. Some effects vanished in different groups, while other improvements only appeared at the follow-up phase. ${ }^{20}$

\section{Institutionalized aged}

So far, there have been few studies investigating this topic, and results are often limited, due to small sample sizes. Reduced attendance and high dropout rates are general problems regarding this population. ${ }^{21}$ Considering the vulnerable population, it is $\operatorname{argued}^{1}$ that PA programs might simply prevent or slow down further deterioration rather than actually improve health. So it is not surprising that there are mixed and non-significant findings on the sustainability of effects of PA interventions in the institutionalized aged.

Regarding high-frequency interventions, Meuleman et al were able to show improvement of function, particularly among individuals with more reduced functional health, but the effects had vanished at the 6- and 12-month follow-up visits. Yet, members of the exercise group had fewer hospital admissions over the 12-month period and had a lower risk of mortality. ${ }^{22}$ Another exercise program improved gait ability in users of residential aged care; this effect was sustainable 3 months after the intervention, with additional long-term effects in balance and lower-limb strength. ${ }^{23}$

With respect to interventions with moderate frequency, Faber et al found positive effects for "prefrail" elderly regarding fall risk reduction at 1-year follow-up; yet fall risk in frail exercisers increased. ${ }^{24}$

We did not come across studies investigating the sustainability of low-frequency interventions.

\section{Summary}

There is little knowledge about the sustainability of effects of PA interventions in the aged - especially concerning users of long-term care. The topic of the study presented in this paper, ie, the sustainability of effects of low-frequency exercise intervention in residential aged care, has previously received little attention.

\section{Study context}

This paper presents the results of a 1-year follow-up study (with data collection in June 2013) researching the sustainability of the effects of a PA intervention in aged care. The initial intervention was part of a comprehensive health promotion project in three units of Austria's largest provider of residential aged care in Vienna, which offers a mix of assisted living (in apartment structures) and nursing care in different types of ward structure. ${ }^{25}$ The initial intervention was organized as a randomized, controlled trial (RCT) study between October 2011 and June 2012. ${ }^{26}$

The intervention was based on a scientific curriculum developed by a group of researchers from Vienna Medical University, specialized in occupational therapy and physiotherapy. ${ }^{27}$ The program was evidence-based and also, explicitly intended to be low-threshold for participants, 
trainers, and the provider organization. This included the following aspects:

- The intervention involved a low exercise frequency (60 minutes, once a week, over 20 weeks)

- It focused on residents' needs and resources rather than maximizing effects on specific dimensions of health (eg, balance, gait speed, or strength)

- The program addressed a variety of dimensions (eg, coordination, balance, strength, endurance, sensorimotor perception, breathing, abilities and skills for managing activities of daily living, and interpersonal skills)

- Participation was facilitated by "reminder" calls and in some cases, organized transportation to the exercise room

- The curriculum specified methodological principles to be considered when implementing the intervention (eg, individualization, repetition and augmentation, reference to everyday life activities, etc) and included examples but left leeway to adapt unit content to the preferences and skills of trainers and participants

- To lower the threshold for the provider organization, the intervention was provided in the form of exercise groups by members of the staff who were already experienced trainers in PA for aged persons. Highly qualified and expensive staff (like physiotherapists or occupational therapists) were to be involved primarily as experts in the role of supervisors and as supportive trainers in units with complex and demanding tasks..$^{26,27}$

The scientific evaluation of the initial intervention had a focus on analyzing the effectiveness of the intervention using an RCT design.

Study participants were recruited following a randomly generated list, by a local project coordinator who was a part of the house staff. Proactive recruitment was done by faceto-face invitation and included argumentation for suitability of the program (also for highly aged persons with chronic illnesses and reduced functional health as well as for hitherto inactive elderly). This approach aimed at involving a broad target group and counteracting a self-selection bias. The eligibility decision was based on expert ratings by physioand occupational therapists who visited the participants in their apartments/rooms. Only residents with major physical impairments (eg, bedridden) or major cognitive impairments were excluded. Due to refusal, reluctance to participate, or to bad functional health status, nearly all 900 residents had to be approached to recruit the planned number of 270 participants for the study. The allocation of the study participants to intervention or control group was done using a random generator, equally distributing the study participants. ${ }^{26}$
The ethics committee of the residential aged care provider officially approved the study protocol. Participants gave their voluntary written consent to be enrolled in the study. ${ }^{26}$

As to results, 222 (intervention group $n=104$, control group $n=118$ ) of 276 participants completed the RCT. The intervention included 14 exercise groups; the size of the exercise groups ranged from seven to 13 persons. Members of the intervention group attended a mean 11.7 of the 20 units of the program; this means an average participation rate of $58.5 \%,{ }^{26}$ which is comparable with similar research. ${ }^{8}$

The main results were as follows:

- The intervention showed a small but significant effect in improving residents' subjective health status, as measured by the EQ-5D ${ }^{28}$

- Occupational performance, as measured by the Canadian Occupational Performance Measure (COPM) ${ }^{29}$ (including activities of daily living), showed a positive, but nonsignificant trend

- No significant results could be shown for objective tests regarding functional health (eg, Timed Up and Go tes ${ }^{30}$ )

- A broad target group could be involved, and there were indications that also formerly inactive persons participated. ${ }^{26}$

Detailed information about the intervention design and effects are the focus of another forthcoming paper.

The objective of the current work, a 1-year follow-up study, was to examine the sustainability of the proven effects of the low-threshold PA intervention on health-related quality of life in residential aged care.

\section{Materials and methods Study design and outcome measures}

The follow-up evaluation took place in June 2013, 12 months after the completion of the trial. To measure the sustainability of the proven effects of the intervention (2011-2012), members of the intervention group were interviewed using a standardized questionnaire, which included measures of health-related quality of life. Occupational performance was added as an outcome measures as there had been a clear positive but non-significant trend. Due to limited resources, there was a decision not to reapply objective functional measures (eg, Timed Up and Go test ${ }^{30}$ ) as there had been no significant effect in the initial study. Scarce resources also led to a decision to focus on the members of the intervention group and to not follow up the control group. Interviewers had a background in occupational therapy, physiotherapy, or nursing science, with practical expertise in work with elderly, and were specifically prepared in a training workshop on how to use the questionnaire. At the 1-year follow-up assessment, 
all 104 residents from the intervention group who had completed the preceding study were invited to take part in the study, by a member of the house staff.

According to the results of the initial study, the main outcome dimension of the follow-up was health-related quality of life (including subjective health status) as measured by EQ-5D. ${ }^{28}$ The EQ-5D is a generic health status measure that includes a descriptive part comprising five subdimensions (mobility, self-care, usual activities, pain/ discomfort, and anxiety/depression). Each dimension is scored on a scale ranging from $1=$ no problems or symptoms to $3=$ serious problems or symptoms. The descriptive profile score can be converted into the EQ-5D index, ranging from 0 (death) to 1 (perfect health). Additionally, the EQ-5D contains a visual analog scale (EQ-VAS), which measures current health state ( scored from $0=$ worst imaginable to $100=$ best imaginable). There is good evidence for reliability, validity, and responsiveness for the EQ-5D in aged people. ${ }^{31}$

Occupational performance was measured using the COPM. ${ }^{29}$ The COPM has confirmed discriminant validity, and test-retest reliability for the item pool has been moderate but has been good for the performance and satisfaction scores. ${ }^{32}$ In our study, the COPM showed poor test-retest reliability on the item pool; subsequently, results on occupational performance were not presented in this paper.

Sociodemographic variables as well as Timed Up and Go test ${ }^{30}$ and a shortened form of the Mini Mental State Examination (MMSE) ${ }^{33}$ which had been applied at the end of the initial study in 2012, were used to analyze the social, functional, and cognitive differences between study participants and dropouts at 1-year follow-up. The Timed Up and Go test ${ }^{30}$ measures the amount of time that it takes for a person to rise from a chair, walk 3 meters, turn around, walk back to the chair, and sit down, while MMSE examines cognitive functions, like arithmetic capacity, memory, and orientation.

\section{Statistical approach}

IBM SPSS Statistics 19 was used for the analytical procedures. The $t$-test and chi-squared test were applied to analyze differences between the study participants and dropouts. General mixed linear models for repeated measures were used to examine the long-term effects concerning metric data. Regarding ordinal data, Friedman tests were used to analyze significance in intertime change. Paired $t$-test and Wilcoxon signed-rank test were applied to determine significant changes between baseline and follow-up.

\section{Results \\ Sample characteristics}

Of 104 participants who had completed the initial intervention, 68 could be included in the 1-year follow-up evaluation, equaling a dropout rate of $34.6 \%$. Main reasons for "drop out" were that participants were not approachable (due to poor health status, including major health complaints or severe cognitive impairment $[n=12]$ or death $[n=11])$. Some participants could not be contacted because of absence in the study period $(n=9)$. Only one person refused to be interviewed. In three cases, no documentation on the reasons for nonparticipation was available.

For a better understanding of this drop-out process, we compared functional and cognitive differences between dropouts and participants of the follow-up study, using extended data results from the initial study postassessment. The Timed Up and Go test ${ }^{30}$ and a shortened form of $\mathrm{MMSE}^{33}$ were analyzed. According to these measures, dropouts had a significant lower MMSE score and more often lived in assisted living or high-intensity nursing units. There was also a clear, though non-significant, trend that dropouts were older and had lower functional capacities. Detailed information concerning sample characteristics is given in Table 1.

As well as information about dropouts, the analysis indicated that participants who initially had mild cognitive impairments were no longer accessible; the follow-up sample thus consists of individuals who were initially not impaired or who were only physically impaired.

\section{Sustainable effects of the PA intervention}

The intervention effects on subjective health status proved to be sustainable; favorable trends were observed for most subdimensions.

Overall time effects analyzed by Friedman tests and generalized linear mixed models indicated significant findings for subjective health status (EQ-5D VAS) and pain/ discomfort (an EQ-5D subdimension). Subsequent detailed analyses were conducted using a Wilcoxon signed-rank test and a paired $t$-test. Subjective health status of participants was still significantly increased compared with the baseline measurement, which equaled a small sustainable intervention effect at 1-year follow-up (Cohen's $d=0.38$; $P=0.02)$. Regarding reported problems with pain/discomfort, the slight increase during the intervention period had been non-significant $(P=0.26)$, but there was a significant overall reduction of reported pain/discomfort when comparing the baseline and 1-year follow-up assessments $(P=0.047)$. 




Figure 1 provides graphics on the longitudinal change in health-related quality of life outcomes. Table 2 shows detailed information regarding descriptive and inferential statistical data.

Taking a closer look at the EQ-5D subdimension scores and their relevance (as can be seen in the average percentage of persons indicating problems over the study period), having pain/discomfort seems to be the most evident problem in this population, followed by mobility problems and depression/ anxiety. Problems with usual activities or self-care are reported to a lesser extent.

Some clear though non-significant dynamics can be observed. Regarding changes in EQ-5D subdimension scores, there was a trend toward stabilization or even improvement in the health dimensions:

- Health-related quality of life, as measured by the EQ-5D Index, showed a continuing but non-significant increase

- As for problems with mobility or usual activities, there was a positive trend reflected in a linear decline in the percentage of individuals reporting problems with mobility and usual activities

- As for self-care, the percentage of participants reporting problems stayed rather stable

- Regarding depression/anxiety, the intervention was associated with a slight but non-significant increase in reported problems, but at the follow-up phase there was a decline of reported problems.

\section{Discussion}

\section{Evidence for sustainable effects of a PA} intervention in the institutionalized aged

At the 1-year follow-up assessment, a large part of the participants of the PA intervention could be reached. Considering age and health status of participants, a dropout rate of $34.6 \%$ for a 1 -year period seems acceptable. Among the participants, those who showed little or no functional or cognitive impairment before the intervention were overrepresented.

For this relatively healthy subpopulation, there was evidence of the sustainability of the effects on health-related quality of life: 1 year after the end of the formal intervention, subjective health status as an overall measure for health-related quality of life was still significantly increased compared with the baseline assessment. This finding has important consequences. Research has shown partially strong associations between subjective health status and mortality, which persist even after adjustments for other health indicators and sociodemographic covariates. ${ }^{34}$ Additionally, health-related quality of life is of special importance in 
A

EQ-5D-subjective health status (range $0-100 ; 100=$ best possible status)

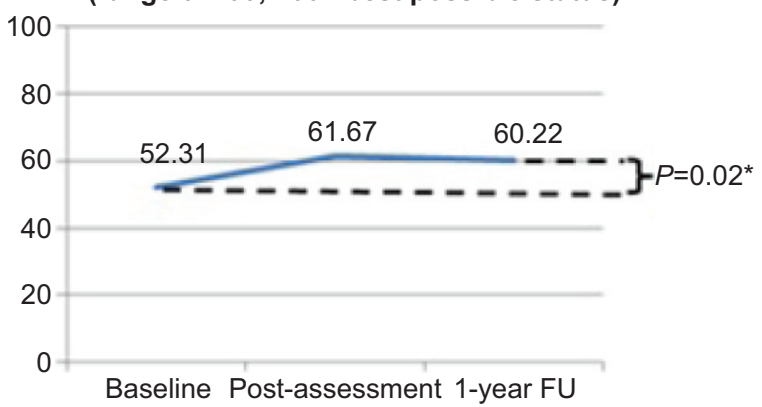

C



E

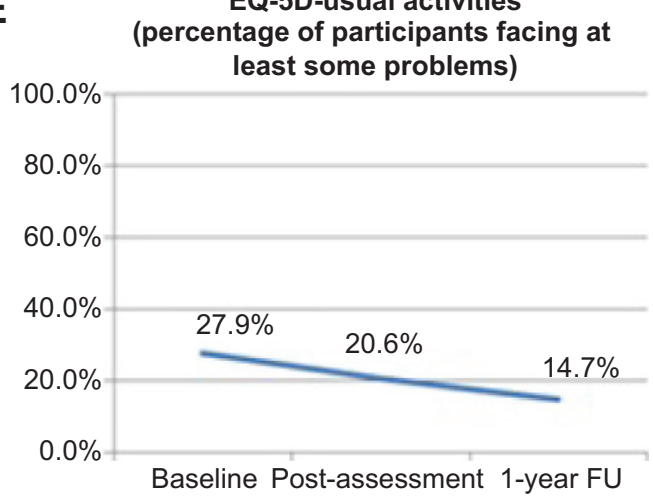

EQ-5D-index

B

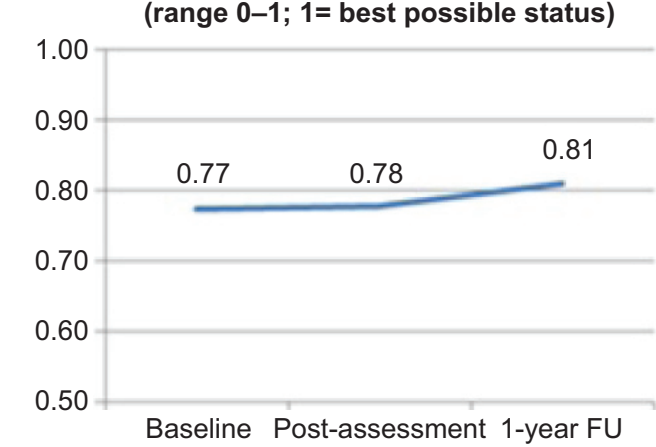

D

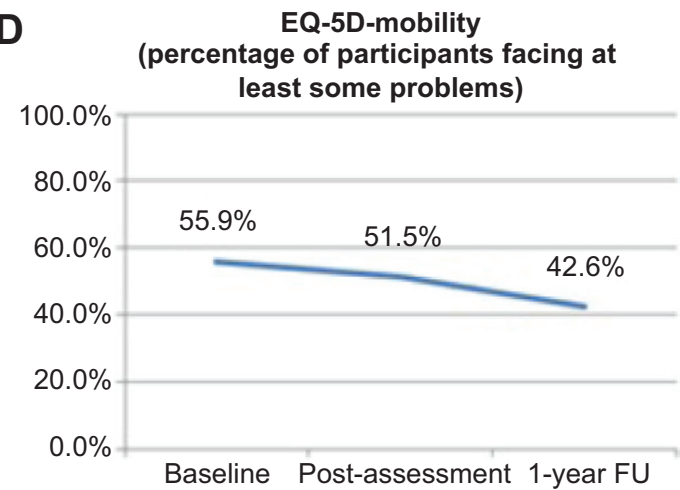

$\mathbf{F}$ EQ-5D-selfcare (percentage of participants facing at least some problems)

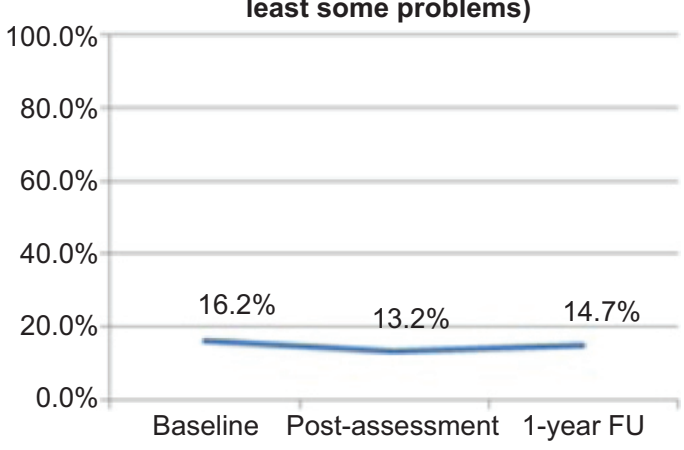

G EQ-5D-anxiety/depression

(percentage of participants experiencing at least some depressive symptoms/anxiety)

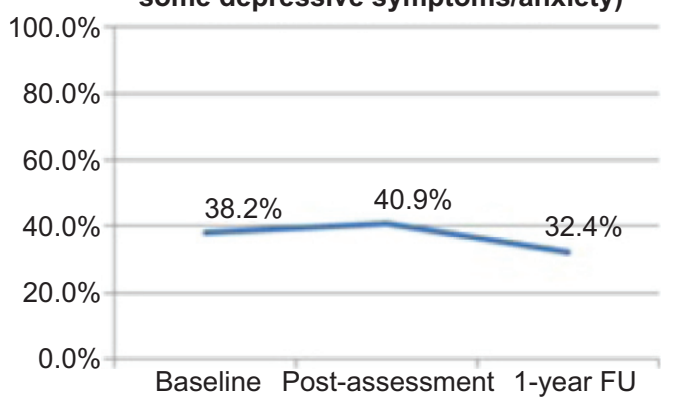

Figure I Graphical analysis of the longitudinal development of the EQ-5D ${ }^{\text {Th }}$ dimensions.

Notes: Intervention group, $N=68$. $* P \leq 0.05$. (A) EQ-5D visual analog scale; (B) EQ-5D index; (C-G) EQ-5D subdimensions. Dotted lines in (A) and (C) are provided to ease visual comparison of changes in scores between different measurement points.

Abbreviations: FU, follow-up; EQ-5D, EuroQol 5 Dimensions. 


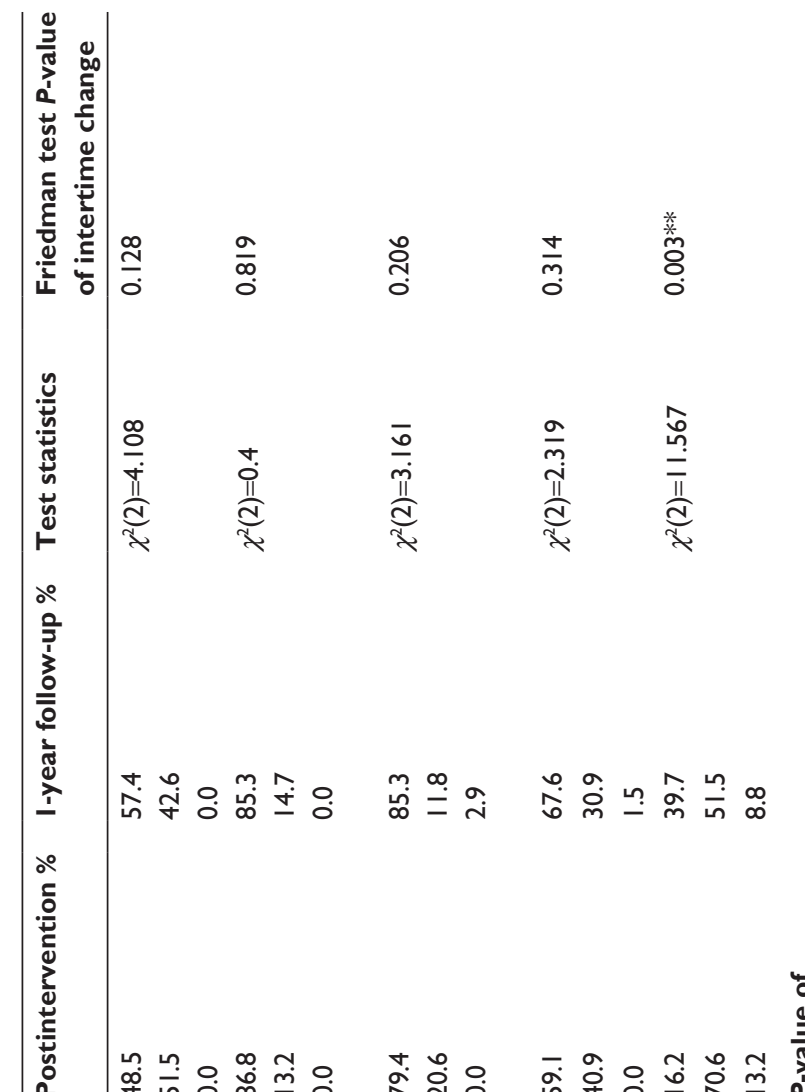

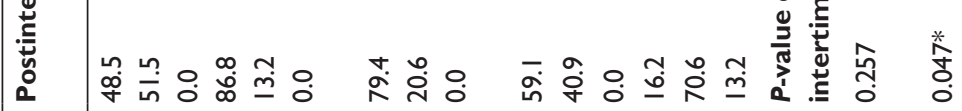

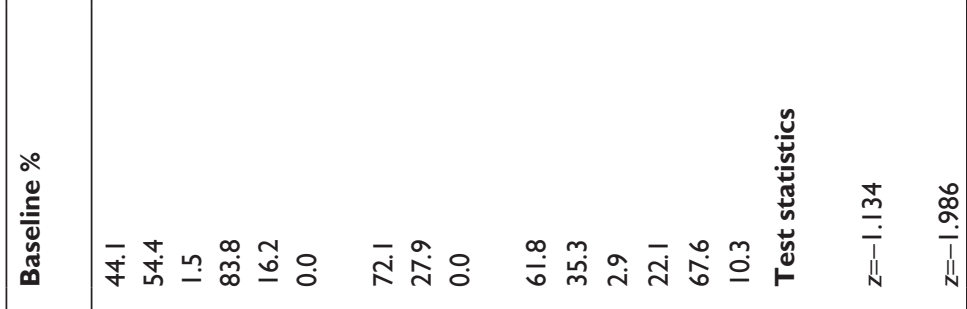

the context of long-term care; it is seen as a relevant and appropriate construct to address quality of care and health of residents in long-term care. The construct comprises multiple aspects of well-being that are affected by progressive health status changes as well as by the health care and social care provided to address these complex and changing needs. By focusing on the individual's rather than health professionals' opinions, the construct emphasizes the principle of "patientcentered care". ${ }^{35}$

As for specific areas of health-related quality of life, positive trends were observed 1 year after the intervention, particularly for problems with mobility and the capacity to perform everyday activities, which were the main target areas of the intervention. As for experiences of pain/discomfort, there was a slight but non-significant increase in reported problems in the intervention period, which might be coincidental or based on a brief sensitization effect during intervention. Yet comparing baseline and follow-up, the results indicate a significant decline concerning the percentage experiencing pain/discomfort. These results support scientific findings that show PA can relieve pain in older adults with chronic pain. ${ }^{11}$

Given the rather low intensity of the intervention, it is likely that at least some of the effects at follow-up were based on individual continuation of training or changes in overall personal PA behavior caused by the intervention. Yet, if there were no changes, initial effects might fade out as time goes by. PA behavior was not recorded in the initial study; consequently, change of PA behavior could not be systematically included in the analysis, and thus we are lacking an empirical basis for attribution of the effect.

A major achievement of this study, beyond proving effects to be sustainable, relates to the character of the intervention (ie, one of relative low frequency and low intensity and not requiring too extensive demands for trainer expertise). This low-threshold PA intervention seems particularly well suited for permanent implementation in aged care facilities, with their limited resources and the limited functional and motivational capacities of the institutionalized highly aged. Although many scientific studies have tried to maximize effects with high-frequency and moderate-tohigh intensity of exercise, ${ }^{14}$ there has also been an example of a low-frequency (such as once a week) program that succeeded in improving specific health outcomes in the aged (eg, strength). ${ }^{36}$ The sustainability of health effects has been researched only for moderate- to high-frequency interventions, but there are few studies, and these indicate mixed findings. It also remains unclear whether or under what conditions a prefrail or frail population, such as the institutionalized aged, can be expected to attend a highly frequent and at least moderately intense exercise program. ${ }^{37,38}$ According to Ferrucci, there are reasonable doubts about the transferability of interventions for the community-dwelling aged to users of residential aged care. ${ }^{39}$ Further, it is likely that intense training programs will bear higher costs and therefore will be less likely to be implemented by residential aged care providers on a routine basis. In this research context, a study that proves the sustainability of the central effect of a low-threshold intervention program that considers the organization's and residents' limited resources seems remarkable.

\section{How can we enhance sustainability of effects of PA interventions in residents of residential aged care?}

As it is likely that sustainability of effects somehow depends on continuation of PA behavior after the completion of the intervention, health services and organizations that provide exercise classes for the aged should employ strategies to enhance further participation. ${ }^{8}$ Regarding the institutionalized elderly, the scientific literature indicates that overall health status is an important challenge in promoting PA. Poor overall health or acute health crises have been stated as main reasons for nonadherence to exercise by the aged ${ }^{8}$ and are frequently mentioned as a barrier to PA behavior in the aged and highly aged, although expectations for improvement of health status, at the same time are referred to as a main motive for participating in PA. ${ }^{7}$ This indicates the importance of creating PA programs that try to systematically address and cope with these problems. Some promising strategies have been identified.

First, it seems evident that effective pain management is a very important basis for the ability to particiapte in PA within the institutionalized aged.

Further, individualization of PA interventions, achieved by tailoring exercise performance (and program frequency) to individuals' needs and resources, can increase the participation of moderately impaired individuals. ${ }^{24,26}$ Costello et al found that intimidation can be an additional barrier for inactive elderly individuals who wish to join established activity programs but who have fears of "slowing down the class" and "not being able to keep up". ${ }^{40}$

Social support has been shown as a facilitating factor for PA in the institutionalized aged. ${ }^{7,41}$ Encouragement (eg, from the family or physician) can be a critical motivator for older adults to exercise. ${ }^{42}$ In this context, proactive recruitment 
(eg, addressing motivational barriers and convincing residents of the usefulness and feasibility of exercising) by provider staff or general practitioners seems relevant to reach a larger group of residents, especially those with impairment or little personal motivation for PA. Understanding the benefits of an exercise program has been shown to be an important motivator for participation in exercise in the aged. ${ }^{8}$

Further, ongoing active support for participation (eg, reminder calls and transportation) from provider organizations seems to be a promising measure to enable and enhance PA participation in residential aged care. ${ }^{15,26}$

Others have recommended that PA interventions for the aged should consider development of self-regulatory skills (eg, goal setting $)^{43}$ or offer larger availability of classes, making it easier to compensate for a missed exercise session in case of illness or other competing activities. ${ }^{37}$

\section{Study limitations}

As in other long-term follow-up studies, the attribution of effects was not distinct in the current study. There was no screening of PA behavior; use of this as a measure to correlate results would have provided indications to attribution of the found effects. Another limitation was the lack of a control group for comparison at follow-up; inclusion would have improved evidence.

Regarding the study population, there are indications that individuals who were initially less functional and/or who were initially not cognitively impaired could be reached at the 1-year follow-up assessment; as a consequence, results are limited to this population.

A further aspect is the absence of systematic screening of pain management in our study, which impeded interpretation of the found effect; inclusion of relevant measurements in future studies is recommended.

Concerning the assessment of occupational performance via the COPM, ${ }^{29}$ some methodological problems occurred in the follow-up that impeded its use for further analysis. Thus, in several cases, activities previously identified as "problematic" were no longer defined in that way, which can be attributed to a wide range of reasons (eg, problems solved, activity could no longer be performed, or some participants did not remember having identified certain activities as problematic). These problems were not rated, thereby causing a selective bias. This result is in accordance with previous evidence that test-retest reliability of the COPM for the item pool is only moderate. ${ }^{32}$ COPM might be appropriate to measure changes in occupational performance in short time periods but not in long-term studies.

\section{Need for research}

Given the small sample sizes of research conducted, the strength of the evidence base for generalized assumptions on the feasibility and effectiveness of PA interventions in the institutionalized elderly is limited, implying the need for further research.

Interventions should be designed to target the population of residential aged care in a representative way, by including formerly inactive, frail, and unmotivated residents. When selecting a trial population in residential aged care, attention should be given to recruitment to avoid selection bias (eg, self-selection bias and restricted eligibility criteria), a problem which is common in this setting. ${ }^{44}$

In addition to program evaluation, researching the underlying mechanisms that enable and enhance residents' participation (in more intense exercise programs as well) might be critical to the effectiveness of PA interventions in this vulnerable group. There is a need for studies to investigate these underlying mechanisms that enable and enhance PA participation, as well as a need to develop and quantify the effects of supportive measures (like proactive support and individualization).

\section{Conclusion}

To our knowledge, this study is the first to research the sustainability of the effects of a low-frequency PA group intervention (1 hour per week, 20 weeks) in residential aged care facilities. This was a 1-year follow-up of the intervention group of a study that had proven effectiveness on health-related quality of life. This study provides indications for the sustainability of effects on health-related quality of life dimensions. Considering the limited resources of residential aged care and limited health condition of residents, it seems likely that provider organizations will be more inclined to implement low-threshold PA interventions and that residents will be more likely to participate in these programs. In this context, the results of the study provide good arguments that this program - combining low threshold with a sound scientific basis - could be implemented on a broad basis. Yet, the sustainability of PA interventions in residential aged care is still quite underresearched; further research is needed to safeguard and deepen findings of this study.

\section{Acknowledgments}

We would like to thank our practice partner KWP, represented by Eva Bader, director for Human Resources and Quality Management, and the local project coordinators Erika Mosor, Ehrentraud Svoboda, and Gertraud Walter 
for their support. We also thank Tanja Stamm and Gudrun Diermayr, from the Medical University of Vienna, who developed the scientific curriculum of the intervention. Vienna Health Promotion Inc., represented by Ursula Hübel, was helpful in sponsoring this research; Michael Zeiler supported the statistical analyses. Financial support for the research and/or publication of this article was given by Vienna Health Promotion, Inc., (WiG) and by the Ludwig Boltzmann Society.

\section{Disclosure}

The authors report no conflicts of interest in this work.

\section{References}

1. Horn A, Brause M, Schäffer D. Bewegungsförderung in der (stationären) Langzeitversorgung. In: Geuter G, Hollederer A, editors. Handbuch Bewegungsförderung und Gesundheit. Bern: Hans Huber; 2012: 305-318. German.

2. Magaziner J, German P, Zimmerman SI, et al. The prevalence of dementia in a statewide sample of new nursing home admissions aged 65 and older: diagnosis by expert panel. Epidemiology of Dementia in Nursing Homes Research Group. Gerontologist. 2000;40(6):663-672.

3. Rodriguez-Blazquez C, Forjaz MJ, Prieto-Flores ME, Rojo-Perez F, Fernandez-Mayoralas G, Martinez-Martin P; Spanish Research Group on Quality of Life and Ageing. Health status and well-being of older adults living in the community and in residential care settings: are differences influenced by age? Aging Ment Health. 2012;16(7):884-891.

4. Physical Activity Guidelines Advisory Committee. Physical Activity Guidelines Advisory Committee Report 2008. Washington, DC: US Department of Health and Human Services; 2008.

5. Mathews AE, Laditka SB, Laditka JN, et al. Older adults' perceived physical activity enablers and barriers: a multicultural perspective. $J$ Aging Phys Act. 2010;18(2):119-140.

6. Lazowski DA, Ecclestone NA, Myers AM, et al. A randomized outcome evaluation of group exercise programs in long-term care institutions. J Gerontol A Biol Sci Med Sci. 1999;54(12):M621-M628.

7. Baert V, Gorus E, Mets T, Geerts C, Bautmans I. Motivators and barriers for physical activity in the oldest old: a systematic review. Ageing Res Rev. 2011;10(4):464-474.

8. Picorelli AMA, Pereira DS, Felício DC, et al. Adherence of older women with strength training and aerobic exercise. Clin Interv Aging. 2014;14(9):323-331.

9. Blair SN, Wei M. Sedentary habits, health, and function in older women and men. Am J Health Promot. 2000;15(1):1-8.

10. Pollock ML, Franklin BA, Balady GJ, et al. Resistance exercise in individuals with and without cardiovascular disease: Benefits, rationale, safety, and prescription. An advisory from the Committee on Exercise, Rehabilitation, and Prevention, Council on Clinical Cardiology, American Heart Association. Circulation. 2000;101(7):828-833.

11. Tse MM, Wan VT, Ho SS. Physical exercise: does it help in relieving pain and increasing mobility among older adults with chronic pain? J Clin Nurs. 2011;20(5-6):635-644.

12. Weuve J, Kang JH, Manson JE, Breteler MM, Ware JH, Grodstein F. Physical activity, including walking, and cognitive function in older women. JAMA. 2004;292(12):1454-1461.

13. Netz Y, Wu MJ, Becker BJ, Tenenbaum G. Physical activity and psychological well-being in advanced age: a meta-analysis of intervention studies. Psychol Aging. 2005;20(2):272-284.

14. Weening-Dijksterhuis E, de Greef MH, Scherder EJ, Slaets JP, van der Schans CP. Frail institutionalized older persons: A comprehensive review on physical exercise, physical fitness, activities of daily living, and quality-of-life. Am J Phys Med Rehabil. 2011;90(2):156-168.
15. Stathi A, Simey P. Quality of life in the fourth age: exercise experiences of nursing home residents. J Aging Phys Act. 2007;15(3): 272-286.

16. Chin A Paw MJ, van Uffelen JG, Riphagen I, van Mechelen W. The functional effects of physical exercise training in frail older people: a systematic review. Sports Med. 2008;38(9):781-793.

17. Pollock RD, Martin FC, Newham DJ. Whole-body vibration in addition to strength and balance exercise for falls-related functional mobility of frail older adults: a single-blind randomized controlled trial. Clin Rehabil. 2012;26(10):915-923.

18. Bird M, Hill KD, Ball M, Hetherington S, Williams AD. The longterm benefits of a multi-component exercise intervention to balance and mobility in healthy older adults. Arch Gerontol Geriatr. 2011;52(2):211-216.

19. Zech A, Drey M, Freiberger E, et al. Residual effects of muscle strength and muscle power training and detraining on physical function in community-dwelling prefrail older adults: a randomized controlled trial. BMC Geriatr. 2012;12:68

20. Freiberger E, Häberle L, Spirduso WW, Zijlstra GA. Long-term effects of three multicomponent exercise interventions on physical performance and fall-related psychological outcomes in communitydwelling older adults: a randomized controlled trial. J Am Geriatr Soc. 2012;60(3):437-446.

21. Au-Yeung SSY, Ho HPY, Lai JWC, Lau RWK, Wong AYL, Lau SK. Did mobility and balance of residents living in private old age homes improve after a mobility exercise programme? A pilot study. Hong Kong Physiotherapy Journal. 2002;20(1):16-21.

22. Meuleman JR, Brechue WF, Kubilis PS, Lowenthal DT. Exercise training in the debilitated aged: strength and functional outcomes. Arch Phys Med Rehabil. 2000;81(3):312-318.

23. Rosendahl E, Lindelöf N, Littbrand H, et al. High-intensity functional exercise program and protein-enriched energy supplement for older persons dependent in activities of daily living: a randomised controlled trial. Aust J Physiother. 2006;52(2):105-113.

24. Faber MJ, Bosscher RJ, Chin A Paw MJ, van Wieringen PC. Effects of exercise programs on falls and mobility in frail and pre-frail older adults: A multicenter randomized controlled trial. Arch Phys Med Rehabil. 2006;87(7):885-896.

25. Krajic K, Cichocki M, Quehenberger V. Health-promoting residential aged care: a pilot project in Austria. Health Promot Int. Epub 2014 March 28

26. Cichocki M, Quehenberger V, Krajic K. "Gesundheit hat kein Alter" Wiener Pilotprojekt zur Erprobung Setting-Orientierter Gesundheitsförderung in der Stationären Altenbetreuung und Pflege. Evaluationsbericht. Wien: LBIHPR; 2013. German.

27. Stamm T, Diermayr G. Curriculum für die interdisziplinäre Intervention "Bewegtes Leben" im Projekt "Gesundheit hat kein Alter". Wien: Gesundheit hat kein Alter; 2011. Available from: http://www. gesundheithatkeinalter.at/Publikationen/Publikationen. Accessed July 29, 2014. German.

28. EuroQol Group. EuroQol - a new facility for the measurement of health-related quality of life. Health Policy. 1990;16(3):199-208.

29. Dehnhardt B, George S, Harth A. Canadian Occupational Performance Measure [German edition]. 4th ed. Idstein: Schulz-Kirchner; 2009. German.

30. Podsiadlo D, Richardson S. The timed "Up \& Go": a test of basic functional mobility for frail elderly persons. J Am Geriatr Soc. 1991; 39(2):142-148.

31. Haywood KL, Garratt AM, Fitzpatrick R. Quality of life in older people: a structured review of generic self-assessed health instruments. Qual Life Res. 2005;14(7):1651-1668.

32. Cup EH, Scholte op Reimer WJ, Thijssen MC, van Kuyk-Minis MA. Reliability and validity of the Canadian Occupational Performance Measure in stroke patients. Clin Rehabil. 2003;17(4):402-409.

33. Haubois G, de Decker L, Annweiler C, et al. Derivation and validation of a short form of the Mini-Mental State Examination for the screening of dementia in older adults with a memory complaint. Eur J Neurol. 2013;20(3):588-590. 
34. Idler EL, Benyamini Y. Self-rated health and mortality: a review of twentyseven community studies. J Health Soc Behav. 1997;38(1):21-37.

35. ZubritskyC,AbbottKM, HirschmanKB, Bowles KH, FoustJB, NaylorMD. Health-related quality of life: expanding a conceptual framework to include older adults who receive long-term services and supports. Gerontologist. 2013;53(2):205-210.

36. DiFrancisco-Donoghue J, Werner W, Douris PC. Comparison of onceweekly and twice-weekly strength training in older adults. Br J Sports Med. 2007;41(1):19-22.

37. Chin A Paw MJ, van Poppel MN, Twisk JW, van Mechelen W. Once a week not enough, twice a week not feasible? A randomised controlled exercise trial in long-term care facilities [ISRCTN87177281]. Patient Educ Couns. 2006;63(1-2):205-214.

38. Rydwik E, Frändin K, Kerstin F, Akner G. Physical training in institutionalized elderly people with multiple diagnoses - a controlled pilot study. Arch Gerontol Geriatr. 2005;40(1):29-44.

39. Ferrucci L. The Baltimore Longitudinal Study of Aging (BLSA): a 50-year-long journey and plans for the future. J Gerontol A Biol Sci Med Sci. 2008;63(12):1416-1419.
40. Costello E, Kafchinski M, Vrazel J, Sullivan P. Motivators, barriers, and beliefs regarding physical activity in an older adult population. $J$ Geriatr Phys Ther. 2011;34(3):138-147.

41. Brenner I, Marsella A. Factors influencing exercise participation by clients in long-term care. Perspectives. 2008;32(4):5-11.

42. Grossman MD, Stewart AL. "You aren't going to get better by just sitting around": physical activity perceptions, motivations, and barriers in adults 75 years of age or older. Am J Geriatr Cardiol. 2003;12(1):33-37.

43. Chao D, Foy CG, Farmer D. Exercise adherence among older adults: challenges and strategies. Control Clin Trials. 2000;21(5 Suppl): 212S-217S

44. Forster A, Lambley R, Young JB. Is physical rehabilitation for older people in long-term care effective? Findings from a systematic review. Age Ageing. 2010;39(2):169-175.
Clinical Interventions in Aging

\section{Publish your work in this journal}

Clinical Interventions in Aging is an international, peer-reviewed journal focusing on evidence-based reports on the value or lack thereof of treatments intended to prevent or delay the onset of maladaptive correlates of aging in human beings. This journal is indexed on PubMed Central, MedLine,

\section{Dovepress}

CAS, Scopus and the Elsevier Bibliographic databases. The manuscript management system is completely online and includes a very quick and fair peer-review system, which is all easy to use. Visit http://www.dovepress. com/testimonials.php to read real quotes from published authors. 